ulcerative lesion. I may not know what "copper color" is; if you speak of a raw-ham color, that means something to me, but I have never seen a copper color in a macular syphilide, nor has the student in my clinic seen it.

\section{AN INTERESTING CASE OF LUPUS VULGARIS.*}

R. R. CAMPBELL, M.D.

I'rofessor of Skin aud Venereal Diseases, Chicago Policlinic; Associate Dermatologist, Staff Cook County Hospital, etc. cHicago.

The patient who furnished the subject of this short paper was referred to me by Dr. Henry Burwash of Chicago. The history of the case is as follows:

Mrs. M. W., aged 53, Canadian; married fifteen years; no children; was never pregnant; had never been robust, but enjoyed fairly good health; never had a blemish of any kind or character on the skin until the age of 48 when, three months after an operation for the removal of some polypoids from the nose, she noticed a small nodule on the left cheek. About this lesion soft papules continued to appear for three years, when ulceration commenced in the original nodule. The ulcerative process spread peripherally until two-thirds of the cheek had become involved in the destructive process, as attested by marked scarring and some ulceration at the time the patient came under my observation. There were also present a nodule on the right cheek and several nodules in the neck and throat, with marked enlargement of the sublingual and submaxillary glands on both sides. These enlarged glands had persisted for over four years, without any evidence or appearance of pus. One brother died of consumption twenty years ago, our patient having cared for him ten months previous to his death. Two aunts, on both the mother's and father's side of the family, died of consumption. The patient's mother and father, however, are still living and enjoying good health.

\section{COMMENTS ON THE CASE.}

The points of special interest in this case, to my mind, are :

1. The age at which the disease developed.

2. The enlarged lymphatic glands.

3. The possible sources of infection, namely, from her tubercular brother, or at the time of the operation on her nose.

4. The differential diagnosis between lupus vulgaris and tuberculosis verrucosa cutis.

Our ideas regarding Iupus vulgaris have undergone radical changes during the past quarter of a century. We have passed through a heated controversy regarding the nature of the disease, and Kaposi and his followers, who contended that lupus vulgaris is not tuberculosis, are, I am convinced, ready to acknowledge that this question is no longer a live issue. Most of us were brought up in the belief that the disease invariably begins in childhood. We have lived to learn that there are exceptions to this rule. The disease may develop at any age of life. Our text-books of to-day are much less positive on this point than they were a few years ago. Elliott ${ }^{1}$ has reported one case and cites five other cases of the disease which developed in adult life. We have become accustomed to looking on lupus vulgaris as true tuberculosis of the skin, and tuberculosis verrucosa cutis as tuberculosis by inoculation. This distinction is a convenience in teaching, but we, as specialists, must recognize the fact that it is far from being accurate. Lupus vulgaris may be produced by inoculation and it

* Read at the Fifty-fourth Annual Session of the American Medical Association, in the Section on Cutaneous Medicine and Surgery, and approved for publication by the Executive Committee: Drs. H. W. Stelwagon, W. L. Baum and J. A. Fordyce. p. 476 . may be produced by a transformation of other forms of tuberculosis.

A few months ago a boy was presented to the Chicago Dermatological Society by Dr. Pardee with a large ulcer on the cheel, from the surface of which luxuriant granulations were growing. The members present agreed on the diagnosis of tuberculosis, but not of lupus. Of the various forms of tuberculosis which were suggested as a possible diagnosis, that of scrofuloderma was the one in which I concurred. Scrofuloderma, as Jadasohn ${ }^{3}$ states, is a subcutancous tubercular deposit, the skin covering which has been destroyed by ulceration, leaving a denuded surface from which fungous granulations spring up. In this case, after treatment with the $x$-rays, the granulations disappeared, leaving a scar. When the case was presented to the society a second time typical lupus nodules were present in the scar tissue. In my judgment it was a case of transformation of scrofuloderma into lupus vulgaris.

Fabry ${ }^{3}$ states that he has observed this transformation of scrofuloderma into lupus vulgaris. He has also seen a tubercular gland suppurate, discharge, the fistula heal and lupus develop in the skin adjacent to the scar of the fistulous opening; where the fistula closes such cases may easily be mistaken for primary lupus vulgaris with secondary glandular enlargement.

He further states that he has seen tubercular dactylitis produce lupus as well as tuberculosis of the bones and joints. Crocker says that Jadasohn reports a case in which lupus developed in a tattoo-mark, caused by wetting the India ink with tubercular saliva; also one in which a tubercular ulcer of the finger in a butcher, caused by inoculation, produced lupus at a point higher up on the extremity.

Dubois Havernith" says: "Inoculation by continuity of tissue is frequently observed at the mouth of fistulæe, occurring in connection with tuberculosis of the joints and bones and lymph glands which have suppurated and opened spontaneously or have been cut into by the surgeon."

In a large number of cases it will be found that the disease has originated in some wound (abrasion or scratches), or in some anterior disease, as erysipelas, eczema, a blister, an irritated pimple and the like, pointing clearly to inoculation. Lupus nodules occurring early in a vaccination scar are presumably the result of inoculation of tubercular virus present in the vaccine.

Havernith cites an instance in which a girl who slept with her sister, who was affected with lupus vulgaris of the left cheek, acquired a lupus of the right cheek, which she habitually laid against her sister's face.

These facts are cited simply to show that because the only explanation which we can offer as to the manner in which our patient contracted the disease, namely, at the time of her operation on the nose or by nursing a brother suffering from pulmonary tuberculosis, does not exclude lupus vulgaris as a diagnosis.

\section{DIAGNOSIS.}

Clinically, we concluded that our case was lupus and not tuberculosis verrucosa cutis for the following reasons :

1. The rapid development of the disease.

2. The fact that the lesions present were not the waxylike lesions of tuberculosis verrucosa cutis.

2. Berliner Klinische Wochenschrift, 1900 .

3. Archiv. fur Dermatologie und Syphilis, vol. xxxix.

4. Diseases of the Skin, third edition, p. 774 .

5 . British Journal of Dermatology, 1891, p. 360 
3. Because the brown spots of beginning lupus were present.

There were no typical velvety, waxy nodules of lupus present at the time the case was under observation, but it is not uncommon to find them absent in typical cases when the patient first comes under observation. The lesions present were all such lesions as may be seen in lupus. We must endearor to recognize and diagnose the atypical as well as the typical cases, and at the same time not lose sight of the fact that we have what may be termed border-line cases.

\section{HISTOLOGY.}

Histologically, the case showed the structure and the deep-seated location of lupus. As is well known, the lupus nodule is deep seated, while the lesion of tuberculosis verrucosa cutis is superficially located.

Stelwagon ${ }^{6}$ shows an excellent cut of tuberculosis verrucosa cutis, taken from one of Bowen's cases, which shows the downward prolongation of the rete pegs and the superficial location of the disease, which was not the microscopic findings in our case.

By inoculation is, to my mind, the most frequent manner of contracting lupus vulgaris in adult life.

The enlargement of the lymphatic glands in connection with lupus may be due to surface infection, but, as Leloir has shown, it may also be due to the presence of tubercle bacilli in the gland. The non-inflammatory character of the enlarged glands in this case, and the length of time they had existed, rather impress me with the opinion that they were tuberculous. The lymphatic glands which were enlarged were situated on each side of the chin in the neck, indicating that absorption had occurred through the superficial facial lymphatics.

That tuberculosis of the skin is contagious in a greater or less degree there can hardly arise any doubt. In view of this fact it is well to follow the suggestions of Neumann, ${ }^{7}$ who believes that inoculation of children and others by persons with tuberculous lesions on the skin or adjacent mucous membrancs is more frequent than is generally recognized. In one place he observed four cases in one year of tuberculous lesions of the penis in children who had been circumeised. Tuberculous lesions of the upper air passages, as well as of the face and mouth, may inoculate others with tubercle bacilli by intimate contact, especially active and passive osculation. He urges scrupulous care in regard to disinfection of articles used by persons with any cutaneous tuberculous affection, scrofuloderma, etc., and regards cutaneous tuberculosis as, in fact, a more threatening source of infection than pulmonary tuberculosis, for the reason that its tuberculous nature is so often unrecognized. Dressings used by persons with tuberculous lesions of the nose, vulva, vagina or uterus, etc., scrofuloderma, miliary tuberculosis and lupus should be burned at once and attendants should frequently disinfect their hands.

414 Marquette Building.

\section{DISCUSSION.}

Dr. J. H. Duncan, St. Louis-From the description of Dr. Campbell's case I could not help thinking there might be a mistake in the diagnosis. I could not make up my mind that it was a typical case of lupus vulgaris, but rather some other tubercular condition of the skin. I learn that there was a difference of opinion as to the character of the case when it was presented to the Chicago Dermatological Society.

Dr. Josepir Zeisler, Chicago-I should like to use this

6. Diseases of the Skin, p. 689 .

7. Neumann: Wiener Klin. Rundschau, vol. xvii, No. 1. occasion to emphasize that dermatologists should be exact in their diagnosis of cases which fall under the generic term of tuberculosis of the skin, pathologically speaking. I long ago joined with those who look on lupus as an absolutely classical manicstation of tuberculosis of the skin. But with others, 1 recognize a great many other forms of tuberculosis of the skin, many of which have been mentioned in this paper and which are clinically totally different from lupus. Yet, we still see a great deal of slipshod use of the term tuberculosis of the skin, which, in the last ten or fifteen years, has become to many what we would call in German "cine Eselsbruicke" (pons asinorum). In other words, it is used by a great many to cover up their unfamiliarity with clinical dermatology when a distinct variety of cutaneous tuberculosis should be specified. Particularly is it necessary, it seems to me, to retain the term lupus for all those forms of tuberculosis which belong to the classical type we have all learned to recognize as lupus, a disease which begins in early life and is characterized by deep-seated, brownish nodules, which may retain their condition for many years. A diagnosis of lupus is often made not in harmony with this clinical picture. The fact Dr. Campbell has pointed out that his patient acquired her trouble at the age of 48 , or something like that, would not necessarily confuse me. I have formerly believed and taught that lupus always begins before puberty, but that can not be maintained at the present time; we certainly see cases of true lupus which begin later in life. I would not question the Doctor's diagnosis on that account. I was somewhat surprised that Dr. Campbell found it necessary to differentiate his case from tuberculosis verrucosa cutis. To my mind tuberculosis verrucosa cutis is distinctly confined, as a rule, to the backs of the hands and fingers, and I could not gather from his description that there was that peculiar wart-like condition or small stalactite prolongations from the skin which I would consider necessary to make that diagnosis. In conclusion, I wish once more to urge the need of retaining the different varieties of tuberculosis of the skin under distinct clinical names rather than to make it too easy for us by grouping all of these cases under the generic term of tuberculosis.

Dr. Henry G. Anthony, Chicago-I agree with Dr. Zeisler that we must maintain very strictly our divisions, and that we must look on lupus as a distinct type to be separated from everything else. Further, I agree with him that it has heretofore been used in a loose manner very frequently.

I had an opportunity of seeing Dr. Campbell's case. It was shown before the Chicago Dermatological Society. Some of those present made a positive diagnosis of tuberculosis ver. rucosa cutis and disputed the diagnosis of lupus. I was interested in the histologic picture the case presented, I therefore obtained from Dr. Campbell some of the tissue and studied it with care, comparing it with a section from tuberculosis verrucosa cutis, and it corresponds with lupus in every way.

Dr. R. R. Campbell, Chicago-In answer to Dr. Duncan's criticism, I tried to clearly set forth that no claim was made that it was a typical case of lupus vulgaris, but that we should be able to recognize and diagnose atypical as well as typical cases; furthermore, that we must recognize that we have borderline cases.

Regarding the suggestion of Dr. Zeisler, I question if tuberculosis verrucosa cutis should come into consideration. It was brought up at the meeting of the Chicago Dermatological Society, and Dr. Zeisler himself at that time suggested that we must look for the canse of the infection, that while he concurred in the fact that it might be a case of lupus, and probably was, yet he would like to know where the infection came from, as it was unusual to find the disease beginning at that period of life. As to tuberculosis verrucosa cutis occurring only on the backs of the finger's, we have exceptions to that rule. I have in my practice now a case of tuberculosis verrucosa cutis of the fingers and over the patella, and several months amo $I$ discharged as cured a case of tuberculosis verrucosa cutis of the buttocks, referred to me by Dr. J. B. Murphy of Chicago, to be treated with the $x$-ray. I think we should be cautious about laying too much stress on the classical seats of diseases. 